\section{Preparation and Mechanical Properties of Polymeric Nanocomposites with Hydroxyapatite and Hydroxyapatite/Clay Mineral Fillers - Review \\ Lenka Pazourková ${ }^{1,2}$, Gražyna Simha Martynková1,2* and Daniela Plachá ${ }^{1,2}$}

${ }^{1}$ Nanotechnology Centre, VSB - Technical University of Ostrava, 70833 Ostrava-Poruba, Czech Republic

2IT4 Innovations Centre of Excellence, VSB - Technical University of Ostrava, 70833 Ostrava-Poruba, Czech Republic

\begin{abstract}
Nanocomposites of hydroxyapatite/polymer and hydroxyapatite/ polymer/clay mineral are studied for pronounced further application in medicine. The ceramic filler influence the mechanical properties of polymeric matrix. This mini review summarizes the influence of hydroxyapatite on the mechanical properties of polymeric matrix, distribution of hydroxyapatite particles in the polymer and also adhesion between ceramic particles and polymer matrix. Very important is size and morphology of filler, because it influences the mechanical properties of polymer. The nanoclays are often used fillers for polymers, since they improve several characteristics of virgin polymer. The combination of hydroxyapatite and nanoclay as the filler for polymers leads to complex improvement of properties and therefore it extends use of these composites.
\end{abstract}

Keywords: Clay mineral; Composite; Hydroxyapatite; Mechanical properties; Polymer

\section{Introduction}

Polymer-ceramic nanocomposites are hybrid organo-inorganic materials. The filler phase is required at nanometer level, it means that at least one dimension of the filler phase is less than $100 \mathrm{~nm}$. Boosted research interest in field of the nanocomposites is observed last decade [1-4] because of remarkable improvement of various nanocomposite properties adding low volume fractions of fillers [3].

*Corresponding author: Gražyna Simha Martynková, Nanotechnology Centre, VSB - Technical University of Ostrava, 70833 Ostrava-Poruba, Czech Republic, Tel: +420 597329546; E-mail: grazyna.simha@vsb.cz

Citation: Pazourková L, Martynková GS, Plachá D (2015) Preparation and Mechanical Properties of Polymeric Nanocomposites with Hydroxyapatite and Hydroxyapatite/Clay Mineral Fillers - Review. J Nanotechnol Nanomed Nanobiotechnol 2: 007.

Received: May 28, 2015; Accepted: June 20, 2015; Published: July 08, 2015
The virgin polymer exhibits poor mechanical properties such as low strength and modulus. Introducing a small amount of ceramic particles into the polymer matrix, we can observe improvement of the properties of polymer [4]. One of the most common filler for polymers, especially for medical uses is hydroxyapatite, which is the most common apatitic calcium phosphate ceramic. The most important calcium phosphates are hydroxyapatite, tricalcium phosphate and dicalcium phosphate. Hydroxyapatite (HAp), $\mathrm{Ca}_{10}\left(\mathrm{PO}_{4}\right)_{6}(\mathrm{OH})_{2}$, is a major component of hard tissues. HAp is very cheap material, which has wide utilization in medical field and also in industry. Synthetic HAp finds many applications mainly in bioceramic [5-8].

Clay minerals are natural materials from phyllosilicate family. They are hydrous aluminium, iron and magnesium silicates with layered structure. Clays have characteristic layered structure contains aluminia octahedral layer and two silica tetrahedral sheets [9]. Polymer/HAp nanocomposite is widely studied materials in recent years. The main advantage is their utilization in medicine, due to improved mechanical properties and also biocompatible properties [10]. The aim of this mini review is to summarize the preparation of polymer/HAp nanocomposites, the influence of HAp filler on mechanical properties of nanocomposite and also the dispersion of hydroxypapatite in polymeric matrix. Polymer/clay mineral composites are widely used in recent years, and also the exploration of this topic is significant. It was many times proven that addition of small amount of clay mineral in to the polymer matrix improves the mechanical properties of polymer [11-13].

Combination of polymer, hydroxyapatite and clay mineral leads to the composite with unique properties and wide range of application, especially in the medicine, since the non-living, inorganic chemical make-up of our teeth and bones is primarily composed of hydroxyapatite (Figure1).

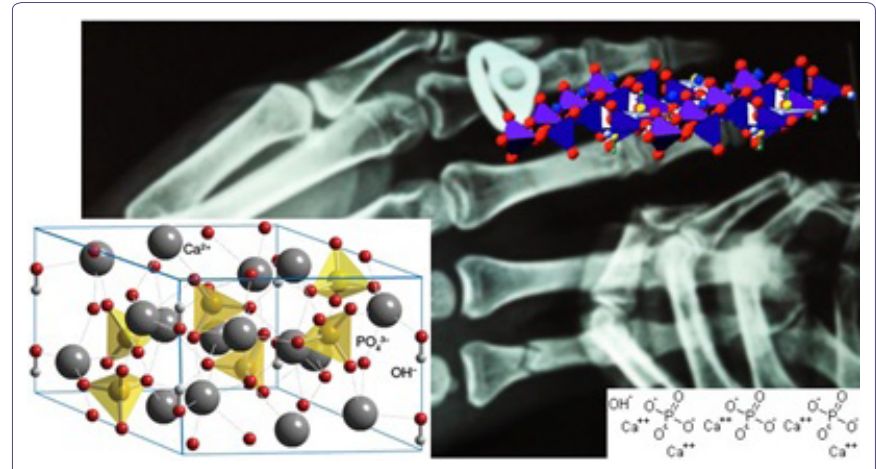

Figure 1: Hydroxyapatite - a building unit of bones and hard tissues.

In this review the main aspects of apatitic calcium phosphates are presented. Focus is here on basic preparation techniques and their limitation, properties of prepared nanocomposites and draft-possibilities of application. 


\section{Preparation of Hydroxyapatite/Polymer Nanocom- posite}

Preparation of HAp/polymer includes several methods. One of these methods include solvent/solution casting method, thermally induced phase separation, electrospinning methods, in situ mineralization of HAp in polymers, electrodeposition and many others figure 2 .

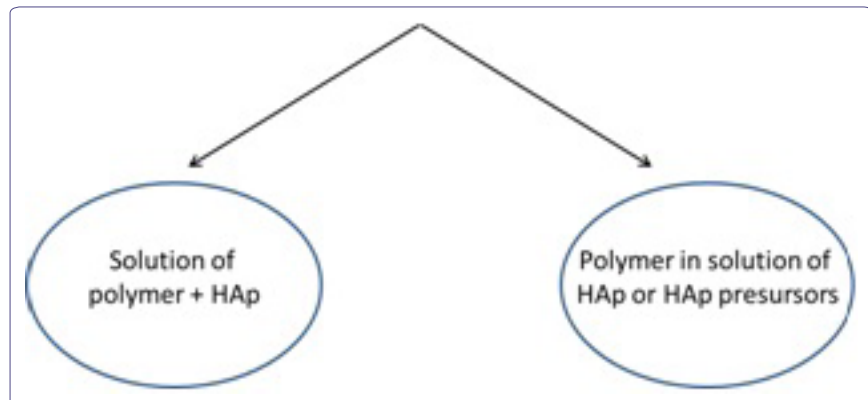

Figure 2: Schema of two basic approaches of nanocomposite preparation.

\section{Solvent/solution casting method}

Solvent casting method figure 3 includes dissolution of the polymer in suitable solvent (organic or inorganic). Then the solution is mixed with additives (inorganic particles, organic matters). The final dispersion is casted in to predefined mold or thin films are prepared and at the end the solvent was allowed to evaporate $[14,15]$. The great advantage of this method is easy preparation and operation without the need of special equipment $[14,16]$. The prepared film often exhibits high flexibility [16].

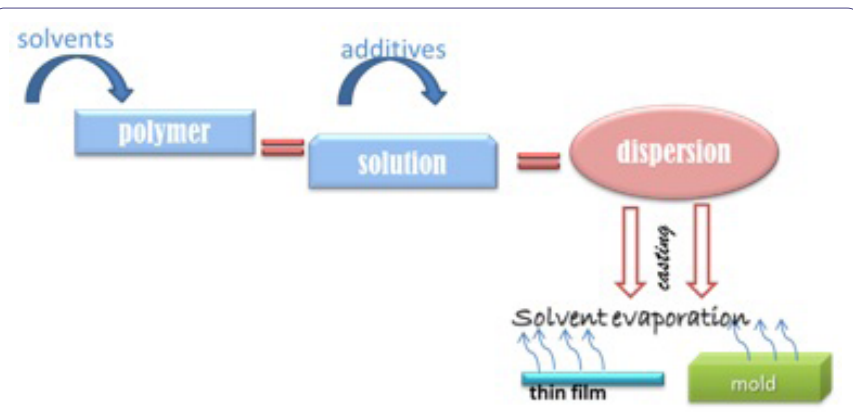

Figure 3: Schema of solvent casting method of nanocomposite preparation.

The advantages of this method are: good dispersion of the filler in the polymer matrix and removal of solvent from final nanocomposite. Combination of solvent casting with another method should solve several problems. For example using freeze drying method for removal of solvent as was reported during preparation of poly (lactic-co-glycolic acid)/nano hydroxyapatite particles by Aboudzadeh et al., [17]. Utilization of ultrasound and different solvent can improve the dispersion of hydroxyapatite in poly (D-,L-lactide) and also prevent aggregation of HAp in polymer matrix [18].

\section{Thermally induced phase separation (freeze-drying method)}

Freeze-drying method, which is known as thermally induced phase separation, consists of formation an emulsion of polymer solution in an organic solvent and water mixture by homogenization. Then the emulsion is rapidly cooled to lock in the liquid state structure. And finally solvent and water is removed by freeze-drying. Materials with porosity more than $90 \%$ and pore size with wide range can be prepared with this method $[19,20]$. The disadvantage of this method is the closed pore structure in final material.

Changing of freeze temperature, ratio and concentration of initial components and type of initial materials (polymer, solvent) strongly influenced mechanical and structural properties of final nanocomposite. Sun et al., [21] prepared multi-layered film of hydroxyapatite and chitosan by this method. Authors studied the influence of different freezing temperature and composition ratio on the mechanical properties of the multi-layered film of hydroxyapatite and chitosan. Mechanical strength of nanocomposite was improved by modification of initial solidification temperature as well as on composition ratio of initial materials. The results also showed that increasing amount of HAp increased the tensile strength and Young's modulus. And authors propose to use this method for preparation films for medical uses.

Zhang et al., [22] studied porous composite of poly (a-hydroxyl acids)/hydroxyapatite prepared by freeze drying method. They investigate that microstructure of the composite foams can be controlled by several parameters, for example by concentration of the polymer solution, amount of the HAp in the composite, the quenching temperature (cooling rate) and the polymer and solvent utilized. The composite foams also exhibit improve mechanical properties in comparison with pure polymer foam.

\section{Electrospinning techniques}

Electrospinning (ES) is a unique, very simple and universal method using electrostatic force to produce fine fibers from polymer solutions or melts (Figure 4).

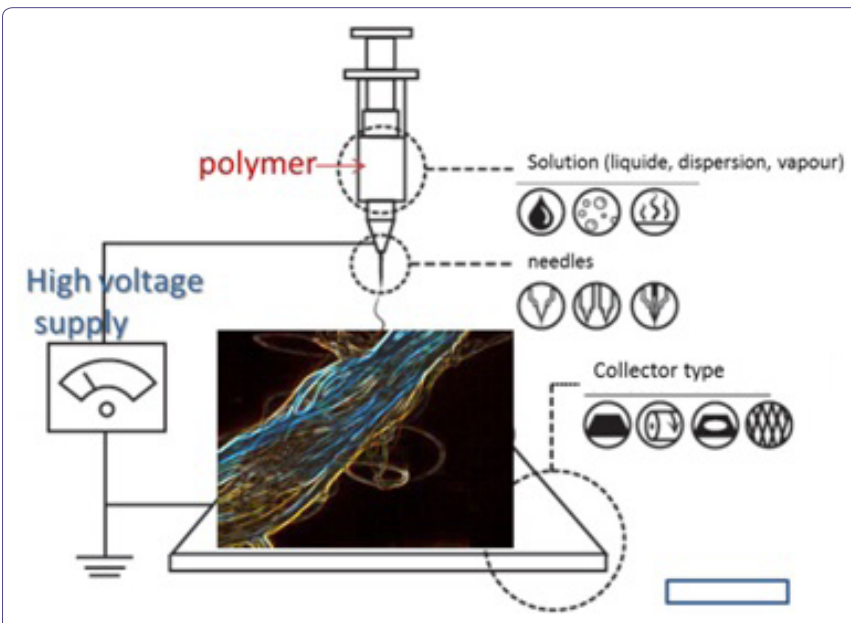

Figure 4: Schema of electrospinning method of nanocomposite preparation.

The produced fibers have a thinner diameter (from $2 \mathrm{~nm}$ to several micrometers) and a larger surface area against fibers prepared by conventional spinning processes. Furthermore, a DC voltage (several tens of $\mathrm{kVs}$ ) is necessary to create the electrospinning. The principle of this method is that between a droplet of polymer solution and grounded target a strong electrical field is applied. When the weaker forces of surface tension of the droplet are overcome by applied electric field, the charged jet of polymer solution is ejected. The jet of polymer is transported and deposited in the target $[23,24]$. The Electrospinning (ES) techniques have two standard setups-vertical and horizontal. Due to expansion of ES, several research groups have advanced improved systems that can produce more complex nanofibrous structures [25,26]. Electrospinning is 
performed at room temperature conditions. Generally, an electrospinning system figure 4 consists of three major parts: a high voltage power supply, a spinneret (e.g., a pipette tip) and a grounded collecting plate-collector (metal screen, plate, rotating mandrel, etc.,) and uses a high voltage source to inject charge of a specify polarity into a polymer solution or melt, which is then speeded towards a collector of opposite polarity [27,28]. Lot of the polymers are dissolved in suitable solvents before electrospinning. It is important to completely dissolve the polymers in solvent to form homogenous polymer solution.

There are several ways how to prepare polymer/HAp nanocomposite. One of them is simple preparation of polymer fiber which is subsequently immersed in special liquid containing HAp particles or its precursors. Fibers prepared by this way show increased hydrophillicity and large surface area, which is suitable for cell adhesion [29]. Another way how to introduce the HAp into polymer fiber is to prepare polymer solution for ES containing HAp nanoparticles. But in this case is important to prevent agglomeration of nanoparticles for example by decreasing of added nanoparticles amount. Addition of appropriate amount of HAp into polymer fiber leads to improve of mechanical properties as elastic modulus, ultimate strength. On the other hand, addition of $10 \mathrm{wt} \%$ of HAp leads to improvement of mechanical properties of final fibers [30]. This method provides several advantages as simplicity in the fabrication of various fiber patterns [12], controlling of fiber morphology by concentrations variation of polymers and initial materials (as nanoparticles, additives). It can be produced fibers from inorganic and also organic materials [23]. The challenge for this method is to find appropriate amount of additives (higher amount leads to decrease of mechanical properties) to established better mechanical properties in contradiction of pure polymers.

\section{In situ mineralization of HAp in polymers}

In situ mineralization is biomimetic process in which mineralization occurs in the close association with the polymer. Principle of this method is crystallization of HAp in the solution in the presence of ionic synthetic polymers. This process will facilitate the occurrence of high degree of interaction and bonding between the organic and inorganic components of the composite which should lead to the better mechanical properties [31,32]. Multiple interactions between in situ HAp and polymer occur. Calcium and phosphorus ions can be trapped in polymer precursor. Several polymers can influence the nucleation, growth and assemble of HAp crystals in its network. This rate of diffusion, chemical and physical properties can be managed by $\mathrm{pH}$ changing. The $\mathrm{pH}$ value can also influenced strength of interaction between polymer and inorganic ions [33].

The advantage of this method is easy preparation and in comparison to other methods. This method produces materials with easily controllable and modifiable microstructure which resembles the topographic features of natural matrix [34]. The size of HAp by adjusting the content of polymer and concentration of $\mathrm{Ca}^{2+}[33]$ as well as the thickness of HAp layer by utilization of proteins deposited on polymer surface [35] can be controlled. As it was mentioned in previous part, in the case of increasing amount of HAp it can lead to improving of mechanical properties [36].

Due to viability to control shape, size, crystal structure and also orientation, the organization of inorganic mineral in polymeric matrix has made biomimetic methods of preparation of HAp composites very interesting [33].

\section{Electrodeposition}

The principle of this process is deposition of particles suspended in the liquid medium by an electric field on the electrode - this is called electrophoretic deposition $[37,38]$. As substrate can be used different materials as metal plates, wires, graphite substrates. The thickness of deposited layer is depending on deposition time [39]. The advantage of electrodeposition technique is precise control of the film thickness, uniformity, deposition rate and low processing temperature. Another advantage is its attractiveness of low equipment cost and starting materials. Electrodeposition is very suitable method, because it can form uniform films on substrates of complicated shape, also impregnates porous substrates and deposits material on the selected areas of substrates. All of these are possible due to using of electric field [40]. Grandfield et al., [41] prepared coating composite of hydroxyapatite-silica-chitosan by electrophoretic deposition. The composite consists of chitosan, hydroxyapatite and silica. The chitosan was used for electrophoretic stabilization and charging of ceramic nanoparticles. The special properties of chitosan, as good binding and film forming, enable the formation of thick coatings in the range of up to $100 \mu \mathrm{m}$. Due to this method is possible to form HAp-silica-chitosan coating by co-deposition of HAp and silica. The advantage of this method is room temperature processing.

\section{Dispersion of Hydroxyapatite in Polymer Matrix}

One of the factors influencing dispersion of particles in the polymer and also its mechanical properties is particle size. Wang et al., [42] observed that HAp particles of average size around $3.3 \mu \mathrm{m}$ exhibit good dispersion and a homogenous distribution in polyethylene matrix due to the compounding process of composites. HAp in higher volume fraction show small amount of agglomerates in the composites, which remained undispersed in the composite. This result is not surprising since $45 \mathrm{vol} \%$ of HAp represents $73 \mathrm{wt}$ $\%$ of HAp of the composite. If it not uses the dispersing agent, small agglomeration of HAp particles in the composite may be expected.

The same author also compared HAp with two different sizes. Wang et al., [43] used two synthetic hydroxyapatite powders and dispersed them in the polymer matrix. The particle sizes of received powders were $4.14 \mu \mathrm{m}$ and $7.32 \mu \mathrm{m}$, respectively. But particle size distribution identified peaks corresponding 3.8 and $6.5 \mu \mathrm{m}$. The monitored parameters were torsional modulus and tensile properties (more Young's modulus, tensile strength and fracture strain). Both strength and modulus of composites significantly increased with increase of hydroxyapatite volume fraction, while fracture strain decrease. Not only amount of hydroxyapatite play role in influencing the mechanical properties. Also particle size can affect changes in the polymer properties. Large HAp particles reduce strength and modulus, but increase ductility of final composite. On the other hand, it was observed increases of torsional modulus, tensile modulus and tensile strength in composites with smaller particles, however it was observed lower failure strain. Between filler and matrix existed only mechanical bond, which was investigated by examination of fracture surface [43].

Kasuga et al., [44] studied modulus of elasticity, bending strength and stress of composite with HAp fibers. The results show that bending strength is almost independent on HAp amount in composite. Bending strength and modulus of elasticity are also influenced by Molecular weight $(\mathrm{Mw})$ of polymer. Polymer used in this work has high value of $\mathrm{Mw}$, the values of bending strength of polymer are also 
high (50-60 MPa). This is comparable with literature reports [45]. Authors also studied the influence of HAp orientation on bending strength, but results do not show significant differences in bending strength after different orientation of HAp fibers. In the case of modulus of elasticity, increasing content of HAp fibers improves effectively modulus of elasticity. Introducing even $20 \mathrm{wt} \%$ of HAp fibers improves modulus of composite. Modulus of PLA/HAp fibers composite containing $70 \mathrm{wt} \%$ of HAp fibers is very high; because it was suggested that modulus of HAp fibers is very high. So it can be concluded that when the polymer with very high Mw (very high modulus) is used as matrix for HAp fibers, the final composite exhibit very high value of moduli. The curves of stress-strain showed that fracture of pure polymer and polymer with $20 \mathrm{wt} \%$ of HAp fibers proceeds gradually beyond maximum stress. So the properties of matrix phase strongly influenced those of composite. Increasing amount of HAp fibers in the composite decreases the maximum strain. From this result can be observed that HAp fiber in composite creates bonds between HAp fibers and polymer [44].

Also HAp whiskers can influence mechanical properties of polymer matrix. Roeder et al., [46] compared effect of HAp whiskers and HAp spherical particles on the mechanical properties of high-density polyethylene matrix. Composites include different amount of filler, from 0 to $50 \mathrm{vol} \%$. Polymer reinforced with HAp whiskers exhibit higher elastic modulus and ultimate tensile strength as compared with composite reinforced by spherical HAp particles. Increasing volume fraction of filler from resulted in improved of mechanical properties (e.g., elastic modulus and tensile stress). Whiskers possessed improved mechanical properties of composites over those filled by spherical particles. It is interesting that composite including HAp whiskers have properties more like that of bones, instead of those filled by spherical HAp. For example, elastic modulus of composites reinforced by 30-50 vol \% of HAp whiskers exhibit values in the range 6-11 GPa, which corresponds to values of cortical bone [46].

The particle size/dimension of HAp (Figure 5) for nanocomposite could play interesting role in improvement of properties. Weia et al., [47] studied influence of HAp Nanoparticles (NHAp) on mechanical properties of the Polylactid Acid (PLLA). The compressive modulus of composite nano HAp/PLLA increased with NHAp content. Pure polymer has compressive modulus $4.3 \mathrm{MPa}$. Adding $30 \%$ of NHAp to the polymer significantly increase the mechanical properties of nanocomposite. The modulus reaches up $8.3 \mathrm{MPa}$, when the ratio between NHAp and polymer is 50:50. Therefore the incorporation of nanoparticles improves mechanical properties of polymer composites.

\section{Modification of Hap}

The properties of polymer/HAp nanocomposites are also influenced by interfacial strength between filler and polymer. The mechanical properties, mainly in tensile strength, are influenced on good adhesion or binding between polymer and HAp, because bad adhesion leads to defect at interface. The important factor affecting the bonding or adherence of two materials (e.g., filler surface, polymer) is wetting. The wetting means addition of special matter on HAp surface and/or polymer. This factor is depending on the hydrophilicity/polarity of the filler and the availability of polymer polar groups. Hydrophilicity is ability of molecule to bond temporary water through hydrogen bonds. The hydrophobic materials interact with themselves and with other substances through van der Waals forces. Therefore, these materials have low or no capacity to form

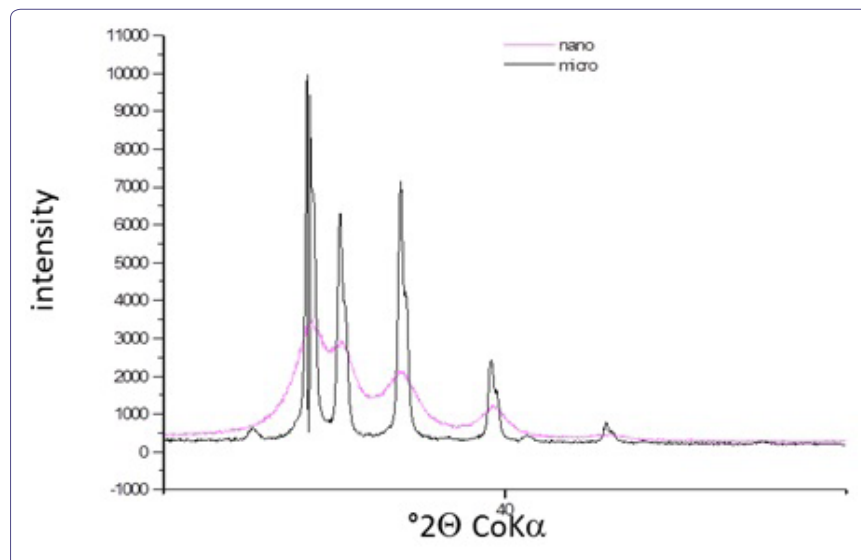

Figure 5: Section of diffraction patterns of nano (diffuse pattern) and micro (sharp pattern) hydroxypatite.

hydrogen bonds [48]. For example the polyethylene is a non-polar and hydrophobic polymer; here exists only mechanical interlocking between HAp particles and polymer matrix in a processed HA/high-density polyethylene composite [42]. If the polymer carries some polarity, it will be achieve a good affinity between HAp and polymer. The adhesion between filler and the polymer could be improved by graft copolymerisation of polar monomer, for example acrylic acid, onto polyolefins [49]. Interfacial adhesion can be also enhanced by using of coupling agents for molecular bonding between a polymer matrix and inorganic fillers. Between polymer and inorganic particles may occur following interactions: materials with strong interactions between two components (covalent, coordination, ionic), weak interactions (van der Waals, hydrogen bonds), or without chemical interactions between two components. Kickelbick et al., [50] classified four types of mutual arrangement of nanoparticles in polymer matrix: 1) inorganic particles embedded in inorganic polymer, 2) incorporation of particles by bonding to the polymer backbone, 3) an interpenetrating network with chemical bonds, and 4) an inorganic - organic hybrid polymer.

Literature gives lot of studies about modification of HAp and polymer for improving adhesion/dispersion of HAp particles in polymer matrix. Wang et al., [51,52] studied two approaches to improve the interfacial condition: the use of silane-treated HAp as the filler and the application of polymer grafting for polyethylene. Authors used two types of commercially prepared hydroxyapatite particles at different amounts. The prepared composites include not only pure hydroxyapatite, but also contain coupling and grafting agents. The results show that using of treated HAp and polymer leads to improve of bonding between this two parts of composite. Increasing amount of HAp leads to improve of mechanical properties as tensile strength, Young's modulus and fracture strain. Additives improved tensile strength of nanocomposites, but they had little bit worse values of Young's modulus. On the other hand, literature suggests that coupling agents improve the dispersion of ceramic fillers in polymer matrices [49] and [53], but extruded HAp/HDPE composites do not exhibit dispersion and distribution of ceramic particles in polymer matrix as suitable as were expected. Introduction of interfacial phase, in this case silane and acrylic acid, leads to new composites with improved properties as ductility and tensile strength, even if they Young's modulus is decreased slightly. Increasing amount of hydroxyapatite results in an increase of Young's modulus and tensile strength as was already stated several times. It was also observed corresponding decrease of fracture strain [52]. 
Another way how to improve dispersion of HAp was studied by Zuo et al., [54]. They compare influence of two polymers (different ratios) on the mechanical properties of final composite including hydroxyapatite. It was investigate that increasing amount of polyethylene affected the properties and microstructure of composite. For example when the composite, including $40 \mathrm{wt} \%$ of HAp, 54 wt $\%$ of polyamide and 6 wt $\%$ of polyethylene, the polyethylene fine granules are dispersed in matrix homogenously and have elastomeric effect on composite. So, the mechanical properties of this composites exhibit good stiffness and elasticity, and are comparable with cortical bone. Composites including $40 \mathrm{wt} \%$ of HAp, $18 \mathrm{wt} \%, 30 \mathrm{wt} \%$ of polyethylene and corresponding amount of polyamide exhibit decrease of mechanical properties. The reason of decrease of mechanical properties can be affected by formation of net structure with many cavities and microcracks. On the other hand, HAp in composite creates hydrogen bonding with polar groups of polyamide - the adhesion between HAp and polyamide is high - which lead to improve of mechanical properties.

Surface modification of HAp improves the distribution of HAp particles at high loading level in the polymer matrix. This process also prevents or delays the de-bonding process and aggregation of HAp particles from the polymer matrix. The commonly used modifiers are organic materials - improve also bonding between HAp and polymer via organic modifier and leads to better surface properties of HAp. Liu et al., [55] studied surface modification of nano-apatite by grafting polymer. Authors' report that there are two ways how to modify the HAp surface by organic molecules:

- The first method includes surface adsorption (adsorption of polymers and proteins).

- The second method is graft organic molecule through covalent bonding to the hydroxyl groups which are available on the crystal surface of HAp.

The surface of the HAp presents hydroxyl group which seem to be reactive. $\mathrm{OH}$ groups of HAp are used for grafting of organic molecules. For example Nishizawa et al., [56] used organic silane coupling agent for modification of calcium phosphate ceramics. Result of their study is that silane coupling agent was covalently bonded to the surface of the ceramics through the reaction with surface hydroxyl groups of the ceramics.

Fang et al., [57] modified surface of HAp whiskers by $\gamma$-aminopropyltriethoxysilane. Modified HAp was added to the poly-L-lactide acid and was compared mechanical properties with pure HAp composite. The whiskers of modified HAp do not exhibit different morphological characteristic. It was studied composites with different amount of HAp whiskers. The comprehensive strength of composite with modified HAp whiskers increase twice compared with pure one. Also comprehensive strength of modified HAp whiskers/polymer was about $47.2 \%$ higher than value of composite filled by non-modified HAp whiskers.

Another way shows direct grafting of polymer on the HAp surface. Liu et al., [55] used polyethylene glycol for modification of HAp. Isocyanate group could react with tree reactive species on the HAp surface (surface adsorbed water, $\mathrm{HPO}_{4}{ }^{2-}$ and $\mathrm{OH}^{-}$). For the successful grafting of polymer removal of adsorbed water is necessary. Authors used nonstoichiometric HAp - they suggested that $\mathrm{HPO}_{4}{ }^{2-}$ is present on the surface of nano-apatite, but on the surface a hydrolysis of $\mathrm{PO}_{4}{ }^{3-}$ occurs. The results do not support the reaction mechanism between $\mathrm{HPO}_{4}{ }^{2-}$ and isocyanate. It was believed that surface hydroxyls groups of HAp have the reactivity towards isocyanate group. Grafting of polyethylene glycol to HAp was not influenced by size and shape of HAp. It was found that grafting reaction takes place only on surface of HAp. Hong et al., [58] investigated grafting of Poly-L-Lactide (PLLA) on the HAp surface. The grafting reaction takes place on the hydroxyl group of HAp by ring-opening polymerization of L-lactide. The reaction took place in the presence of the catalyst. As was describe by Liu et al., [55], Hong et al., [58] used dry HAp to prevent formation of free PLLA (may occur in the presence of water). The particles of grafted PLLA/HAp exhibit good dispersion in the chloroform and form good suspension. On the other hand, non-grafted HAp particles do not form completely precipitate suspension in the chloroform. The composite containing $5 \mathrm{wt} \%$ of non-grafted HAp particles is apt to agglomerate. On the other hand, the grafted HAp particles exhibit uniformly distribution and make homogenous composite.

So it can be concluded, that grafting of HAp particles improve dispersion in polymer matrix. Grafting also prevent agglomeration of HAp particles. The mechanical properties of composite are also influenced by dispersion of HAp particles in polymer matrix. Composites including only pure HAp have properties as brittle materials. The improvement of elongation at break between composite including grafted HAp is two times higher than for pure HAp. Tensile strength of grafted HAp/polymer composite exhibit high values about 54-58 MPa depending on the amount of added grafted HAp particles (10-25 wt \%). On the other hand, composite including pure HAp particles shows monotonously decrease of tensile strength with increasing amount of HAp particles. Presence of grafted nanoparticles improves the toughness and tensile strength. These nanoparticles initiate mass of crazing which can consume great portion of fracture energy and prevent from further developing of defects. The covalent bonding between PLLA grafted HAp and polymer matrix is associated with penetrating of PLLA chains grafted on HAp particles into the PLLA matrix and subsequent entangle with the PLLA chains of the matrix mix. This is the result of improved toughness and ductility. In the case of tensile modulus can be seen that the addition of 1-10 wt \% of HAp powders to the polymer matrix does not change the tensile modulus of composite evidently. But the tensile modulus can be improved by adding of 10-20 wt \% HAp nanoparticles [58].

\section{Adhesion between polymer and hydroxyapatite}

Further aspect which influences mechanical properties of HAp/polymer composites is adhesion between inorganic particles and polymer matrix [59]. The adhesion between HAp and polymer are evaluated by binding energy [60]. The weakest parts of composites are internal interfaces which provide places for nucleation of cracks leads to premature failure. The bond strengths of hybrid biomaterials are critical parameters necessary to controlled them. Molecular modeling can briefly show us the adhesion between HAp and polymer. Zhang et al., [60] studied the molecular dynamic simulation on the interaction between polymers and hydroxyapatite with and without coupling agent. Authors used following polymers: Polyethylene (PE), Polyamide (PA) and Polylactic Acid (PLA). The binding energies of PA/HAp and PLA/HAp are much higher than that of PE/HAp. This can be attributed to the large number of polar groups in PA and PLA groups. The simulation shows that the surface of (110) HAp has higher binding energy with polymers than other HAp surfaces. On the other hand, the binding energy of polymer to 
the HAp is also different and depends on the type of polymer. The PA and PLA have much higher binding energy to HAp than PE. But the addition of coupling agent can improve the interactions between $\mathrm{PE}$ and HAP, but this trend is not arisen in the case of PA/HAp and PLA/HAp interfaces.

Neuendorf et al., [59] investigated adhesion between HAp and several polymers [poly (L-lactide), poly (D-lactide), poly (DL-lactide), 75/25 polylactide/polyglycolide, 50/50 polylactide/poylglycolide and poly ( $\varepsilon$-caprolactone)]. Contact between polymers and HAp exhibit low contact angle $\left(\leq 60^{\circ}\right)$. The poly $(\varepsilon$-caprolactone) shows the highest value of contact angle $\left(62^{\circ}\right)$, on the other hand the lowest contact angle is associated with poly(D-lactide) $\left(16^{\circ}\right)$. Physical interactions control the adhesion between calcium phosphates and biopolymer. Bending strength of polymer/HAp was in the range from 9 to $25 \mathrm{MPa}$. The highest value of bending strength exhibits composite of HAp and polylactide, which was $23 \mathrm{MPa}$. On the other hand the lowest value of bending strength shows poly ( $\varepsilon$-caprolactone)/HAp composite. The formation of physical bond at the organic/inorganic interface is confirmed by measured bond strengths $\sigma_{f}$. For polymer/ceramic interfaces is common physical bonding [61], ceramic grafting is frequently used to enhance adhesion in hybrid composites. It is interesting that poly (L-lactide) and poly (D-lactide) have similar value of bond strengths to HAp, whereas poly (DL-lactide)/HAp represents the bond 20\% stronger and this bond is almost three times stronger than poly ( $\varepsilon$-caprolactone)/HAp bond. The clearly decrease of adhesion between polylactide and HAp occur in the case of addition of polyglycolide. It can be concluded that all polymers exhibit low contact angle on the ceramic [59].

Adhesion between HAp and polymer should be improved for example by increasing temperature during preparation of polymer/HAp nanocomposite [62] or addition of coupling agent [60], which also improve dispersion in polymer matrix as was mentioned previously. This process highly improves compressive strength of final composite [62].

\section{Hydroxyapatite/Clay Mineral/Polymer Composite}

The preparation of this composite includes two main methods: wet precipitation and electrophoretic deposition. Amount of studies about this composite are limited, nonetheless several studies have been carried out. The mechanical properties have not been studied yet. Polymer composites with clay minerals and hydroxyapatite find the utilization mainly in medicine $[63,64]$.

\section{Wet precipitation}

Wet precipitation is often used method for preparation HAp modified clay mineral: Ambre et al., [63] prepared composite which consists of hydroxyapatite, modified clay mineral and chitosan/polygalacturonic. Apatite was formed in situ in the modified clay where hydroxyapatite in the clay exhibits differences in its lattice structure as compared to the ex situ hydroxyapatite. The interactions between modified clay and HAp were observed, which was confirmed also by another study [65]. The ions present in the apatite were possibly mineralized in modified clay. The authors found that modified clay bears some charge in the crystal structure due to precipitation of apatite. Polymer films containing in situ prepared hydroxyapatite on modified clay showed that molecular level interactions existed between the polymer polyelectrolyte and the in situ $\mathrm{HAp} /$ clay.
Kati et al., [66] synthesized nanocomposite consisted of chitosan, montmorillonite and hydroxyapatite, unmodified Na-montmorillonite, hydroxyapatite (prepared by wet precipitation) and chitosan were used. Authors compared the properties of $\mathrm{HAp} / \mathrm{MMT} /$ chitosan, MMT/chitosan and HAp/chitosan composite. The results from XRD analysis reveal that an intercalated structure was achieved with an increase in d-spacing of MMT. FTIR study shows significant interaction between chitosan, MMT and HAp. From the TGA analysis was investigated that adding of inorganic particles (MMT and HAp) to the chitosan improves the thermal stability of the composite. Authors further found that MMT particles are better dispersed in composite of chitosan/MMT/HAp than in the chitosan/MMT composite. The composite of these three components exhibit enhanced nanomechanical properties compare to composite of chitosan with only MMT or HAp, respectively. Nanoindentation tests found that there is a significant increase in the elastic modulus of composites over the pristine polymer. Chitosan/MMT composite has elastic moduli of $\sim 8 \mathrm{GPa}$, the addition of HAp tends to increase the value of the $9 \mathrm{GPa}$. Nanomechanical properties are improved not only by addition of MMT but also by HAp.

\section{Electrophoretic deposition}

Deen et al., [63] prepared monolayer film of chitosan/hydroxyapatite/halloysite by Electrophoretic Deposition (EPD). EPD was used for deposition of halloysite nanotubes and fabrication of composite chitosan-HNT and chitosan-HNT-hydroxyapatite films. Chitosan was used as a dispersing and charging agent for both HAp and halloysit nanotubes, which allowed to the formation of chitosan-hydroxyapatite-halloysite monolayers, films of graded composition and multilayer laminates, containing different layers.

Deen et al., [67] studied electrophoretic deposition of composite halloysite nanotube-hydroxyapatite-hyaluronic acid films. The films were prepared by electrophoretic deposition. The method is based on the use of the natural hyaluronate biopolymer as a dispersing and charging agent for halloysite, HAp and film forming agent for the fabrication of the composite film. Because the suspension of halloysite is unstable, it showed fast sedimentation. So, no EPD was achieved from such suspension. To improve the stability of the suspension and allowed the formation of anodic deposits the sodium, hyaluronate was added. It was assumed that dissociation of Sodium Hyaluronate ( $\mathrm{HYNa}$ ) lead to the formation of anionic species HY. Then occurs adsorption of $\mathrm{HY}^{-}$on the halloysite particles and passed a negative charge for anodic EPD. The composite films prepared by this method exhibit crack free structure. From the SEM analysis were investigated, that increasing amount of halloysite nanotube in the solution increases the amount of this nanotubes in the deposits. This material is also promising for the fabrication of biomedical implants with improved biocompatibility and corrosion protection of metallic implants.

\section{Conclusion}

As we can see from the text, the mechanical properties of polymer matrix are strongly influenced by addition of hydroxyapatite. The properties of composites are also affected by the dispersion of hydroxyapatite in the polymer matrix and adhesion between hydroxyapatite and polymer. There are many methods of preparing these composites and there are also many factors, which have to be controlled for receiving a composite with desired properties. For preparation of HAp/polymer composites are crucial several 
Citation: Pazourková L, Martynková GS, Plachá D (2015) Preparation and Mechanical Properties of Polymeric Nanocomposites with Hydroxyapatite and Hydroxyapatite/Clay Mineral Fillers - Review. J Nanotechnol Nanomed Nanobiotechnol 2: 007.

parameters, as preparation method, utilization of suitable solvent and also grafting agent for good dispersion of $\mathrm{HA}$ in polymer matrix and also for improvement of adhesion between HAp and polymer.

The novel composite of polymer/clay mineral/hydroxyapatite shows properties, which can be used in medical application. The studies about influence of properties by dispersing filler (hydroxyapatite and clay mineral) in polymer matrix have not been performed yet. For future work will be interesting to observe influence of clay minerals on the mechanical properties of final polymer nanocomposite.

\section{Acknowledgements}

This work was supported by projects: Ministry of Education, Youth and Sport of Czech Republic SP2015/59 and IT4 Innovations Centre of Excellence project reg.no.cz.1.05/1.1.00/02.0070.

\section{References}

1. Šupová M, Simha Martynková G, Barabaszová K (2011) Effect of Nanofillers Dispersion in Polymer Matrices: A Review. Science of Advanced Materials 3: 1-25.

2. Martynková GS, Valásková M (2014) Antimicrobial nanocomposites based on natural modified materials: a review of carbons and clays. J Nanosci Nanotechnol 14: 673-693.

3. Sinha Ray S, Okamoto M (2003) Polymer/layered silicate nanocomposites: a review from preparation to processing. Prog in Pol Sci 28 : 15-39.

4. Mittal V (2010) Optimization of Polymer Nanocomposite Properties. WILEY-VCH Verlag GmbH \& Co. KGaA, Weinheim, Germany.

5. Kutikov AB, Song J (2013) An amphiphilic degradable polymer/hydroxyapatite composite with enhanced handling characteristics promotes osteogenic gene expression in bone marrow stromal cells. Acta Biomater 9: 8354-8364.

6. Swetha M, Sahithi K, Moorthi A, Srinivasan N, Ramasamy K, et al. (2010) Biocomposites containing natural polymers and hydroxyapatite for bone tissue engineering. Int J Biol Macromol 47: 1-4

7. Suchý T, Rýglová Š, Balík K, Smetana K, Šupová M, et al. (2013) Biological evaluation of polydimethylsiloxane modified by calcium phosphate nanoparticles for potential application in spine surgery. Science of Advanced Materials 5: 484-493.

8. Šupová M, Simha Martynková G, Sucharda Z (2011) BIOAPATITE MADE FROM CHICKEN FEMUR BONE. Ceramics-Silikáty 55: 256-260.

9. Bergaya F, Theng BKG, Lagaly G (2006) Handbook of Clay Science. In: Developments in Clay Science. Elsevier Ltd, Singapore.

10. Pielichowska K, Blazewicz S (2010) Bioactive polymer/hydroxyapatite (nano) composites for bone tissue regeneration. In: Akihiro Abe, Karel Dusek, Shiro Kobayashi (eds.). Biopolymers. Springer, Germany. Pg no: 97-207.

11. Lvov Y, Abdullayev E (2013) Functional polymer-clay nanotube composites with sustained release of chemical agents. Progress in Polymer Science 38: 1690-1719.

12. Ganigar R, Rytwo G, Gonen Y, Radian A, Mishael YG (2010) Polymer-clay nanocomposites for the removal of trichlorophenol and trinitrophenol from water. Applied Clay Science 49: 311-316.

13. Kiliaris P, Papaspyrides CD (2010) Polymer/layered silicate (clay) nanocomposites: An overview of flame retardancy. Progress in Polymer Science 35: 902-958.

14. Sun F, Zhou H, Lee J (2011) Various preparation methods of highly porous hydroxyapatite/polymer nanoscale biocomposites for bone regeneration. Acta Biomater 7: 3813-3828.

15. Siemann $U$ (2005) Solvent cast technology-a versatile tool for thin film production. In: Stribeck N, Smarsly B (eds.). Scattering Methods and the Properties of Polymer Materials. Springer Berlin Heidelberg, Heidelberg, Germany. Pg no: 1-14.
16. Kim SH, Lim BK, Sun F, Koh K, Ryu SC, et al. (2009) Preparation of High Flexible Composite Film of Hydroxyapatite and Chitosan. Polym Bull 62: 111 118.

17. Aboudzadeh N, Imani M, Shokrgozar MA, Khavandi A, Javadpour J, et al. (2010) Fabrication and characterization of poly (D,L-lactide-co-glycolide)/hydroxyapatite nanocomposite scaffolds for bone tissue regeneration. J Biomed Mater Res A 94: 137-145.

18. Deng X, Hao J, Wang C (2001) Preparation and mechanical properties of nanocomposites of poly ( $\mathrm{D}, \mathrm{L}$-lactide) with Ca-deficient hydroxyapatite nanocrystals. Biomaterials 22: 2867-2873.

19. Ma PX (2004) Tissue engineering. Encyclopedia Of Polymer Science and Technology. John Wiley \& Sons, Hoboken, New Jersey, USA.

20. Liu X, Ma PX (2004) Polymeric scaffolds for bone tissue engineering. Ann Biomed Eng 32: 477-486.

21. Sun F, Lim BK, Ryu SC, Lee D (2010) Preparation of multi-layered film of hydroxyapatite and chitosan. Materials Science and Engineering: C 30: 789794.

22. Zhang R, Ma PX (1999) Poly(alpha-hydroxyl acids)/hydroxyapatite porous composites for bone-tissue engineering. I. Preparation and morphology. J Biomed Mater Res 44: 446-455.

23. Yarin AL, Koombhongse S, Reneker DH (2001) Bending instability in electrospinning of nanofibers. J Appl Phys 89: 3018.

24. Chew SY, Wen Y, Dzenis Y, Leong KW (2006) The role of electrospinning in the emerging field of nanomedicine. Curr Pharm Des 12: 4751-4770.

25. Kidoaki S, Kwon IK, Matsuda T (2005) Mesoscopic spatial designs of nano and microfiber meshes for tissue-engineering matrix and scaffold based on newly devised multilayering and mixing electrospinning techniques. Biomaterials $26: 37-46$.

26. Stankus JJ, Guan J, Fujimoto K, Wagner WR (2006) Microintegrating smooth muscle cells into a biodegradable, elastomeric fiber matrix. Biomaterials 27 : 735-744.

27. Liang D, Hsiao BS, Chu B (2007) Functional electrospun nanofibrous scaffolds for biomedical applications. Adv Drug Deliv Rev 59: 1392-1412.

28. Sill TJ, von Recum HA (2008) Electrospinning: applications in drug delivery and tissue engineering. Biomaterials 29: 1989-2006.

29. Ito Y, Hasuda H, Kamitakahara M, Ohtsuki C, Tanihara M, et al. (2005) A composite of hydroxyapatite with electrospun biodegradable nanofibers as a tissue engineering material. J Biosci Bioeng 100: 43-49.

30. Yang D, Jin Y, Ma G, Chen X, Lu F, et al. (2008) Fabrication and characterization of chitosan/PVA withhydroxyapatite biocomposite nanoscaffolds. Journal of Applied Polymer Science 110: 3328-3335.

31. Kato K, Eika Y, Ikada Y (1997) In situ hydroxyapatite crystallization for the formation of hydroxyapatite/polymer composites. Journal of Materials Science 32: 5533-5543.

32. Yang D, Jin Y, Zhou Y, Ma G, Chen X, et al. (2008) In situ mineralization of hydroxyapatite on electrospun chitosan-based nanofibrous scaffolds. Macromol Biosci 8: 239-246.

33. Li J, Zhu D, Yin J, Liu Y, Yao F, et al. (2010) Formation of nano-hydroxyapatite crystal in situ in chitosan-pectin polyelectrolyte complex network. Materials Science and Engineering: C 30: 795-803.

34. Li D, Xia Y (2004) Electrospinning of Nanofibers: Reinventing the Wheel? Advanced materials 16: 1151-1170.

35. lijima K, Sakai A, Komori A, Sakamoto Y, Matsuno H, et al. (2015). Control of biomimetic hydroxyapatite deposition on polymer substrates using different protein adsorption abilities. Colloids and Surf B Biointerfaces 130: 77-83.

36. Sinha A, Das G, Sharma BK, Roy RP, Pramanick AK, et al. (2007) Poly(vinyl alcohol)-hydroxyapatite biomimetic scaffold for tissue regeneration. Materials Science and Engineering: C 27: 70-74. 
37. Sarkar P, Nicholson PS (1996) Electrophoretic Deposition (EPD): Mechanisms, Kinetics and Application to Ceramics. Journal of the American Ceramic Society 79: 1987-2002.

38. Zhitomirsky I (1998) Electrophoretic and electrolytic deposition of ceramic coatings on carbon fibers. Journal of the European Ceramic Society 18: 849856.

39. Pang X, Zhitomirsky I (2005) Electrodeposition of composite hydroxyapatite-chitosan films. Materials Chemistry and Physics 94: 245-251.

40. Zhitomirsky I, Gal-Or L, Kohn A, Hennicke HW (1995) Electrochemical preparation of $\mathrm{PbO}$ films. Journal of Materials Science Letters 14: 807-810.

41. Grandfield K, Zhitomirsky I (2008) Electrophoretic deposition of composite hydroxyapatite-silica-chitosan coatings. Materials Characterization 59: 61-67.

42. Wang M, Porter D, Bonfield W (1994) Processing, characterization, and evaluation of hydroxyapatite reinforced polyethylene composites. Brit Ceram $T$ 93: 91-95.

43. Wang M, Joseph R, Bonfield W (1998) Hydroxyapatite-polyethylene composites for bone substitution: effects of ceramic particle size and morphology. Biomaterials 19: 2357-2366.

44. Kasuga T, Ota Y, Nogami M, Abe Y (2001) Preparation and mechanical properties of polylactic acid composites containing hydroxyapatite fibers. Biomaterials 22: 19-23.

45. Ozeki E (1996) Characteristics of poly (I-lactide) as biodegradable plastics. Shimadzu-hyouron (in Japanese) 53: 1-8.

46. Roeder RK, Sproul MM, Turner CH (2003) Hydroxyapatite whiskers provide improved mechanical properties in reinforced polymer composites. J Biomed Mater Res A 67: 801-812.

47. Wei G, Ma PX (2004) Structure and properties of nano-hydroxyapatite/polymer composite scaffolds for bone tissue engineering. Biomaterials 25: 47494757

48. Chow TS (1998) Wetting of rough surfaces. Journal of Physics: Condensed Matter 10: 445 .

49. Chiang WY, Yang WD (1988) Polypropylene composites. I. Studies of the effect of grafting of acrylic acid and silane coupling agent on the performance of polypropylene mica composites. Journal of Applied Polymer Science 35: 807-823.

50. Kickelbick G (2003) Concepts for the incorporation of inorganic building blocks into organic polymers on a nanoscale. Prog Polym Sci 28: 83-114.

51. Wang M, Deb S, Bonfield W (2000) Chemically coupled hydroxyapatite-polyethylene composites: processing and characterisation. Mater Lett 44: 119124.

52. Wang M, Bofield W (2001) Chemically coupled hydroxyapatite-polyethylene composites: structure and properties. Biomaterials 22: 1311-1320.
53. Han CD, Sandford C, Yoo HJ (1979) Effects of titanate coupling agents on the rheological and mechanical properties of filled polyolefins. Polymer Engineering \& Science 18: 849-854.

54. Zuo Y, Li Y, Li J, Zhang X, Liao H, et al. (2007) Novel bio-composite of hydroxyapatite reinforced polyamide and polyethylene: Composition and properties. Materials Science and Engineering: A 452-453: 512-517.

55. Liu Q, de Wijn JR, de Groot K, van Blitterswijk CA (1998) Surface modification of nano-apatite by grafting organic polymer. Biomaterials 19: 1067-1072.

56. Nishizawa K, Toriyama M, Suzuki T, Kawamoto Y, Yokugawa Y, et al. (1995) Surface modification of calcium phosphate ceramics with silane coupling agents. Chem Soc Japan 1: 63-67.

57. Fang Z, Feng Q (2014) Improved mechanical properties of hydroxyapatite whisker-reinforced poly(L-lactic acid) scaffold by surface modification of hydroxyapatite. Materials Science and Engineering: C 35: 190-194.

58. Hong Z, Qiu X, Sun J, Deng M, Chen X, et al. (2004) Grafting polymerization of L-lactide on the surface of hydroxyapatite nano-crystals. Polymer 45 6699-6706.

59. Neuendorf RE, Saiz E, Tomsia AP, Ritchie RO (2008) Adhesion between biodegradable polymers and hydroxyapatite: Relevance to synthetic bone-like materials and tissue engineering scaffolds. Acta Biomaterialia 4: 1288-1296.

60. Zhang H, Lu X, Leng Y, Fang L, Qu S, et al. (2009) Molecular dynamics simulations on the interaction between polymers and hydroxyapatite with and without coupling agents. Acta Biomaterialia 5: 1169-1181.

61. Wu S (1982) Polymer interface and adhesion. CRC Press, Florida, USA.

62. Ignjatović N, Tomić S, Dakić M, Miljković M, Plavšić M, et al. (1999) Synthesis and properties of hydroxyapatite/poly-L-lactide composite biomaterials. Biomaterials 20: 809-816.

63. Ambre A, Katti KS, Katti DR (2011) In situ mineralized hydroxyapatite on amino acid modified nanoclays as novel bone biomaterials. Materials Science and Engineering: C 31: 1017-1029.

64. Deen I, Pang X, Zhitomirsky I (2012) Electrophoretic deposition of composite chitosan-halloysite nanotube-hydroxyapatite films. Colloids and Surfaces A: Physicochemical and Engineering Aspects 410: 38-44.

65. Roul J (2012) Design and characterization of novel biodegradable polymer-clay-hydroxyapatite nanocomposites for drug delivery applications. Asian Journal of Biomedical and Pharmaceutical Sciences 2.

66. Katti KS, Katti DR, Dash R (2008) Synthesis and characterization of a novel chitosan/montmorillonite/hydroxyapatite nanocomposite for bone tissue engineering. Biomed Mater 3: 034122.

67. Deen I, Zhitomirsky I (2014) Electrophoretic deposition of composite halloysite nanotube-hydroxyapatite-hyaluronic acid films. Journal of Alloys and Compounds 58: 531-534. 